\title{
Improving handover from intensive care to ward medical teams with simple changes to paperwork
}

Jonathan Messing

Great Western Hospital, United Kingdom

\begin{abstract}
Medical handover has been highlighted by the General Medical Council[1] as a critical step in patient care, ensuring continuity of care, patient safety, and enabling efficient multidisciplinary functioning. Handover between doctors on the intensive care team and the ward teams in the Great Western Hospital on step down was evaluated by assessing discharge summaries and patient notes, and by following up discharged patients. Handover was found to be present only in the minority of patients and documented in none.

Simple changes were made to discharge paperwork in the form of a prompt and documentation of to whom handover was made, as well as the creation of space in the daily review sheets for patients with outstanding handover to be completed. The initial audit findings were presented at a local meeting to remind staff of the importance of handover. These simple modifications brought the handover rates up to $100 \%$ $(n=12)$. The rates of documentation of handover also rose from $0 \%$ to $100 \%$. This quality improvement project serves to demonstrate that carefully targeted, simple changes to practice in identified critical areas can produce dramatic as well as legally and ethically required results in a very short space of time.
\end{abstract}

\section{Problem}

Handover from the intensive care team to ward teams on patient step down was noted to be incomplete in several significant cases in the Great Western Hospital in Swindon, United Kingdom. The receiving ward teams were occasionally forced to ask for clarification of plans and opinions in the days following discharge, but answering these questions proved to be difficult since the discharging physician not always present and notes were transported with the patient.

Examples of this included the decision not to operate further in a cranioplasty patient which was not fully documented, as well as the decision not to intervene in a patient with an ST segment elevation myocardial infarct. To assess this objectively, an audit was carried out to quantify the proportion of discharged patients handed over.

\section{Background}

It is stated in Good Medical Practice: "You must contribute to the safe transfer of patients between healthcare providers...you must share all relevant information with colleagues involved in your patients' care within and outside the team, including when you delegate care"[1], while the National Patient Safety Agency highlighted that, "handover of care is one of the most perilous procedures in medicine, and when carried out improperly can be a major contributory factor to subsequent error and harm to patients."[2] It was stated at local induction to the intensive care unit that patients must be handed over to ward teams upon discharge. Difficulties in completing this in a timely manner including out of hours discharges and differing shifts meant that a significant proportion of patients were being handed over inadequately.

\section{Baseline measurement}

Patients discharged over a fortnight were followed up to assess discharge and handover. Electronic notes were consulted to document time of completion of internal discharge summary. Paper notes were inspected for documentation of verbal handover and receiving teams were asked if they received one:

$-100 \%(n=9)$ of patients had an electronic discharge summary prior to discharge

- $33 \%(n=3)$ of patients had a verbal handover to ward staff

$-0 \%$ of verbal handovers were documented.

See supplementary file: ds5381.docx - "A comparison of old and updated discharge summary templates"

\section{Design}

Interventions included raising the issue to highlight its importance and asking nursing staff to prompt doctors when a patient is being discharged. During presentation of initial audit the intensive care unit staff were consulted regarding what they thought would most help them remember handover. The template for discharge paperwork was modified to include a new line prompting documentation of to whom and at what time verbal handover was made, and the daily handover sheets were modified to include an area for patients discharged out of hours and not yet handed over.

\section{Strategy}


PDSA cycle 1: The initial audit was presented to the department at local meeting and met with approval. Some doctors were not aware of the requirement for handover of patients and appreciated it being brought to general attention. A revised template for discharge summaries was submitted which included a prompt for doctors asking to whom and at what time was handover made. Patients discharged out of hours were left on the handover sheet in italics to highlight that verbal handover had not yet been made and needed doing in hours. After the first week following presentation of data documented handover rates remained low but had started to rise to $17 \%(n=6)$. Electronic discharge summaries were completed in a timely manner throughout the course of this quality improvement project and the rates of those are not recorded here for clarity.

PDSA cycle 2: Leaving patients' names on the handover sheet for handover the following day often did not include sufficient information for accurate handover. Doctors responsible for discharge out of hours were asked to update the handover sheet with all relevant information so someone unfamiliar with the patient could complete handover the following morning. The discharge summary template could not be updated without consultant and administration approval so this was obtained and resubmitted. Handover rates after a week rose slowly to $27 \%(n=11)$.

PDSA cycle 3: The discharge summary template was updated but intensive care doctors complained that finding the contact details for the new team proved difficult. It was mentioned that they were only informed of generic teams rather than specific doctors who were taking over care. A list of pager numbers for the different members of each team was compiled and displayed in doctors' workrooms. Documented handover rates with the new discharge summary and pager numbers rose to $80 \%(n=5)$.

PDSA cycle 4: The template was further updated for aesthetic reasons and to allow it to fit on a single side of paper. As discharge paperwork was being completed days before discharge at a time when the receiving team was unknown, completion of documentation of to whom handover was given to was sporadic. Doctors were reminded that they needed to ensure paperwork was complete and with the patient upon discharge, but the template was adjusted to allow completion with an unknown receiving team initially. Handover rates remained at $80 \%(n=5)$.

PDSA cycle 5: All changes were successfully in place with positive feedback. Further highlighting of the issue informally to doctors helped bring documented handover rates to $100 \%(n=12)$ after five weeks of initial audit presentation. One of the doctors on the unit was nominated "handover champion" and volunteered to ensure compliance remained high and pass on their responsibilities when they rotated.

\section{Results}

Upon re-audit within two months of the initial audit it was found that $100 \%(n=12)$ of patients had an electronic discharge summary completed prior to discharge as well as $100 \%$ with a fully documented verbal handover.
See supplementary file: ds5380.png - "A graph demonstrating the maintained success in discharge summary completion and improvements made in documented verbal handover"

\section{Lessons and limitations}

This audit cycle demonstrates the effectiveness of simple modifications at no cost to the trust. This problem was largely due to lack of awareness of the issue so straightforward changes including a local presentation and basic prompts in paperwork were enough to create the impetus for change. Challenges included bureaucracy behind the templates and obtaining contact numbers for the ward teams but with the support of senior staff these were easily overcome. These changes should be sustainable as long as the discharge paperwork template changes are maintained and this should improve continuity of care for patients and multidisciplinary team-working for staff. The most effective intervention seemed to be the modification to the discharge summary template and making pager numbers readily available as this brought handover rates up from $17 \%$ to $80 \%$ in a single week.

\section{Conclusion}

Simple modifications can have a massive effect and improve experiences for both patients and staff. Although these alterations have not been tested in other departments it is likely these findings are transferable to all other areas of care where handover and discharges are important.

\section{References}

1. General Medical Council [Internet]. UK: Good Medical Practice; Communication, partnership and teamwork; Continuity and coordination of care. 2013. Available from: http://www.gmc-uk.org/guidance/good_medical_practice/con tinuity care.asp

2. British Medical Association [Internet]. Safe Handover: Safe Patients. 2006. Available from:

http://bma.org.uk/-/media/files/pdfs/practical\%20advice\%20 at\%20work/contracts/safe\%20handover\%20safe\%20patient s.pdf

\section{Declaration of interests}

Nothing to declare.

\section{Acknowledgements}

The author would like to acknowledge the staff of the intensive care unit in the Great Western Hospital, particularly Dr Tony Pickworth and Dr Malcolm Watters for their support in this project.

\section{Ethical approval}

Ethical approval was not sought for this project as it was deemed 
an improvement study and not a study on human subjects. 